\title{
Simulation Study on the Flow Field of Muffler Array in Air-cooling Platform Based on Porous Media Model
}

\author{
Peng Xie ${ }^{1}$, Bin Zhang ${ }^{3}$, Xiao-Kuan Lee ${ }^{2}$, Zhong-Xu Kang ${ }^{2}$ \\ ${ }^{1)}$ Beijing Key Laboratory of Environmental Noise and Vibration. Beijing Municipal Institute of Labour Protection, \\ Beijing 100054, China \\ ${ }^{2)}$ State Environmental Protection Engineering Center for City Noise \& Vibration Control. Beijing Municipal Institute of Labour \\ Protection, Beijing 100054, China \\ ${ }^{3)}$ Beijing Municipal Institute of Labour Protection, Beijing 100054, China
}

\begin{abstract}
Porous medium theory is introduced to simplify the muffler array installed in air- cooling platform in this paper. According to the definition of porous medium, the discussion and analysis on geometric structure and flow field characteristics of muffler array show that, the using of porous medium equivalent model for the muffler array is reasonable. The characteristic parameters of porous medium equivalent model are obtained by the measurement and simulation methods. Furthermore, the whole simulation model of air-cooling platform is built with the porous medium equivalent model of muffler array. Then, the effect of muffler array structure on the performance of air-cooling platform is studied with the flow field numerical simulation. The results show that, for the case without environment wind, the air flow of air-cooling platform with muffler array is well distributed, except for the less of air flow; for the case with environmental wind, the installing of muffler array effectively improve the orderliness of the intake air, which is benefit for fans' working. Meanwhile, the increasing of the intake air distance of air-cooling platform caused by the muffler array is benefit for controlling the reflux of hot air. The numerical simulation analysis on flow field of air cooling platform with muffler array can provide assistance for the design and improvement of muffler array to reduce the air-cooling platform's noise.
\end{abstract}

Keywords - Air-cooling Platform, Porous Medium, Muffler, Flow Field, Numerical Simulation

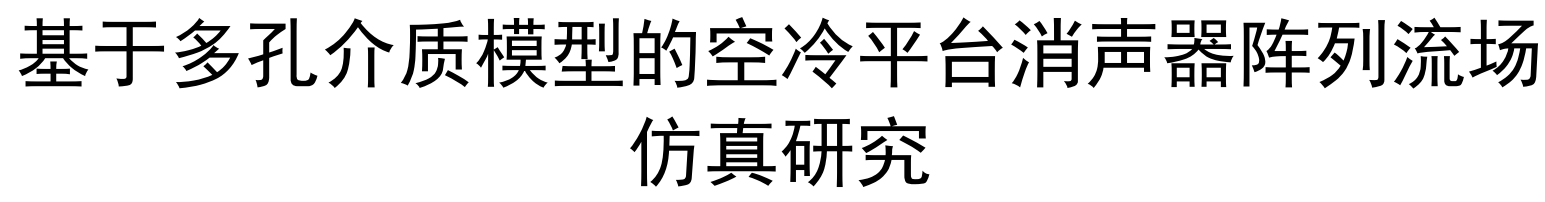

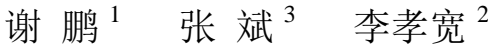 \\ 1) 中国环境噪声与振动北京市重点实验室, 中国 北京 100054 \\ 2) 中国国家环境保护城市噪声与振动控制工程技术中心，中国 北京 100054 \\ 3) 中国北京市劳动保护科学研究所，中国 北京 100054
}

摘 要 本文介绍片了多孔介质理论在空冷平台阵列消声器简化模型中的应用。根据多孔介质的定义, 对消声器阵列的几何特征 进行讨论, 并展开对消声器阵列模型流场特征的分析, 论证多孔介质模型等效消声器阵列模型的合理性; 进而利用实验和仿真方法研 究确定多孔介质模型特性参数, 并建立多孔介质等效消声器阵列的局部模型。而后, 通过对空冷平台流场的仿真计算, 研究了某种消 声器阵列结构对空冷平台性能的影响。结果表明, 在无环境风影响的情况下, 将消声器阵列加入空冷平台后, 流量分布较为均匀, 有 少部分流量损失。在有环境风的情况下, 消声器阵列对空冷平台进口气流有较好的整流效果, 有效改善了由于风速引起的进口气流无 序性, 对风机工作有利。通过对空冷平台的流场数值模拟分析, 可以为消声器阵列的设计和改进提供数据支持已达到降低空冷平台噪 声的目的。

关键词 空冷平台, 多孔介质, 消声器, 流场, 数值模拟 


\section{1. 研究背景}

随着电力工业的迅速发展, 火力发电厂不断增加大容 量, 同时消耗大量水资源, 由于我国内陆地区的水资源严 重匮乏，因此空冷技术被广泛应用于火力发电厂。

直接空冷平台是我国火电厂使用最多的冷却系统设 备, 它由若干风机提供风源, 风机运行的噪声对周边环境 造成很大影响, 安装消声器是控制空冷平台噪声辐射的有 效措施, 但是在目前调研过的文献中还没有查阅到关于在 空冷平台中吊装大型消声器阵列用以降噪的先例, 尤其是 使用数值仿真方法研究消声器阵列对空冷平台流场影响的 案例。

本文将使用 36 组大型消声器阵列吊装于空冷平台进 风口出, 由于其体积庞大且结构复杂, 对于原结构模型进 行建模网格数庞大, 普通计算机无法完成正常计算, 因此 提出使用多孔介质等效消声器阵列模型的数值模拟方法并 加以论述。

\section{2. 消声器阵列的多孔介质等效模型}

通过研究表明, 气体通过消声器组群的流动是典型的 多孔介质-纯流体耦合流动[1] (如图 1 所示)。

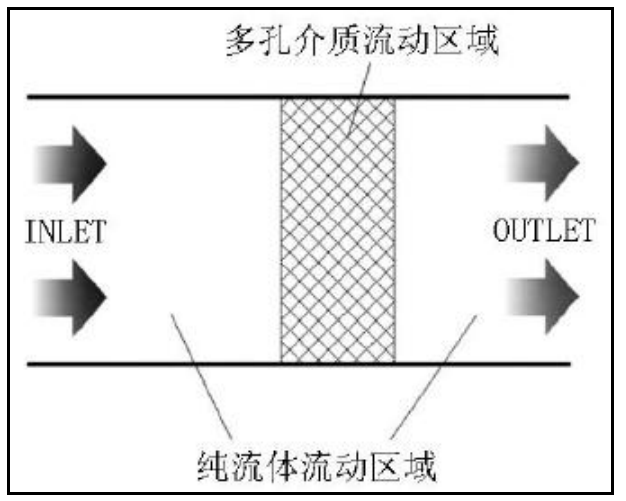

图 1 多孔介质纯流体耦合流动区域模型

对于不可压缩流体, 其 Navier-Stokes 方程的向量形式 为[2]:

$$
\frac{d \bar{V}}{d t}=\bar{f}-\frac{1}{\rho} \operatorname{gardp}+v \nabla^{2} V^{-}
$$

本文使用多孔介质模型等效消声器阵列, 并在模型中 对标准流动控制方程组的动量方程中加上额外的动量源项 来表示多孔介质，其运动方程如下:

$$
\frac{d \bar{V}}{d t}=\bar{f}-\frac{1}{\rho} \operatorname{gardp}+v \nabla^{2} V^{\top}+S_{i}
$$

式 2 中的 $S_{i}$ 就是额外加上的动量源项, 此动量源项由
两部分组成, 一部分是粘性损失项, 另一项是惯性损失项 [3]。

$$
S_{i}=-\left(\sum_{j=1}^{3} D_{i j} \mu V_{j}+\sum_{j=1}^{3} C_{i j} \frac{1}{2} \rho|V| V_{j}\right)
$$

式中, $S_{i}$ 是坐标 $\mathrm{i}$ 方向 $(\mathrm{x}, \mathrm{y}$, 或 $\mathrm{z})$ 动量方程中的动 量源项, 源项第一项 $\sum_{j=1}^{3} D_{i j} \mu V_{j}$ 为粘性损失项, 第二项 $\sum_{j=1}^{3} C_{i j} \frac{1}{2} \rho|V| V_{j}$ 为惯性损失项; $\mathrm{D}$ 和 $\mathrm{C}$ 为预先规定的矩阵系 数; $|V|$ 是速度值; $\mu$ 为动力粘性系数。在多孔介质单元中, 动量损失对于压力梯度有贡献, 压降和流体速度（或平方 根) 成比例, 这也是多孔介质模型动量方程中存在动量源 项（汇）的主要原因[4]。

而对于介质中存在规律状均匀分布的多孔区域, 其为 各向同性时, 多孔介质中流动的流体可以由式 (2) 简化为:

$$
S_{i}=-\left(\frac{\mu}{\alpha} V_{i}+C_{2} \frac{1}{2} \rho|V| V_{i}\right)
$$

式中: $\alpha$ 是指渗透性; $1 / \alpha$ 为粘性阻力系数; $C_{2}$ 是指 惯性阻力系数。

如果通过多孔介质的层流流动中, 压降和速度成正比, 常数 $C_{2}$ 可以忽略为零。方程 (3) 中忽略对流加速以及扩 散, 多孔介质模型可简化为 Darcy 定律:

$$
\nabla P=-\frac{\mu}{\alpha} \vec{V} \text { 即 } V=-\frac{\alpha}{\mu} \frac{d p}{d s}
$$

在此情况下介质在 $\mathrm{x}, \mathrm{y}, \mathrm{z}$ 三个方向的厚度 ( $\left.\Delta n_{x}, \Delta n_{y}, \Delta n_{z}\right)$ 是指多孔区域的实际尺寸。因此如果模 型的大小与实体的尺寸不一样的话, 一定要调整 $1 / \alpha$ 的取 值[5]。

多孔介质等效模型的建立需要两个重要参数: 粘性系 数和惯性阻力系数, 图 2 给出的就是确定多孔介质等效模 型参数的确定流程图。

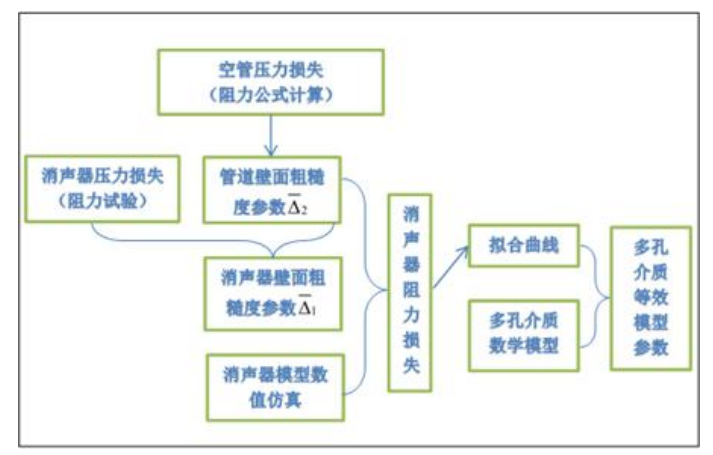

图 2 多孔介质等效参数确定流程图 
根据上述方法得出模型参数, 并参考多孔介质等效模 型的定义现建立与消声器组群宏观尺寸相同的多孔介质等 效模型如图 3 所示, 并进行数值仿真计算[6]。

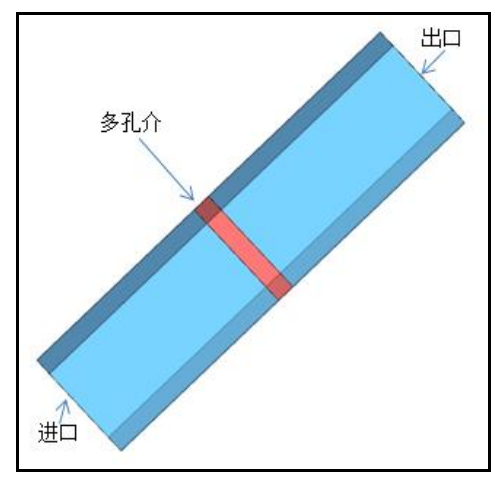

图 3 多孔介质等效模型

\section{4. 空冷平台流场仿真分析}

整体模型如图 4 所示, 三维模型包括平台、支柱及部 分建筑物的结构, 尺寸按照相关设计图纸建模。消声器阵 列采用上节简历多孔介质模型等效, 其位置处于风机进风 口下侧, 四周封闭。

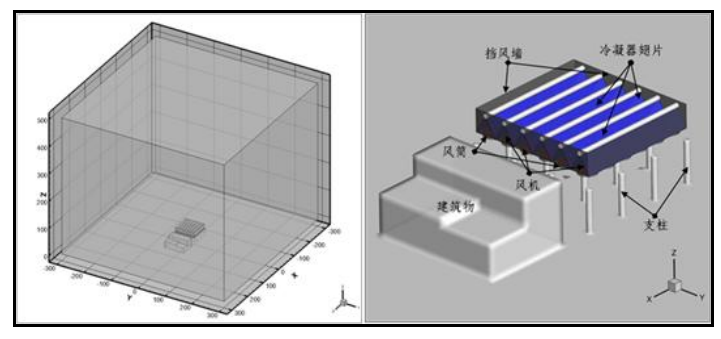

图 4 空冷平台三维模型

由于气流流速低, 采用工况为不可压空气, 但考虑到 空气加热后具有一定的上浮效果, 采用 Boussinesq 密度模 型, 该模型只考虑温度变化产生的密度变化; 方程组离散 格式为迎风离散格式, 采用 SIMPLE 算法求解方程组; 流 动模型采用标准的 湍流模型; 由于计算域大, 空气体积大, 计算中考虑气体的重力效应; 迭代过程中除了监测各残差 曲线外, 同时监测风筒下表面压力、风机体积流量等物理 量, 当残差小于 10-4 且监测量基本不变时认为计算收玫。

\section{1 无环境风条件}

表 1 显示, 在无风条件下, 此消声器阵列方案的平均 压力与无消声器相比损失为 $12.4 \mathrm{~Pa}$, 体积流量为设计总流 量的 $93.0 \%$, 与无消声器流量相比损失 $520.9 \mathrm{~m} / \mathrm{s} 3$, 约为 $3 \%$ 。
表 1 结果分析表

\begin{tabular}{|c|c|c|c|}
\hline & 无消声器 & 加装消声器 & 平均损失 \\
\hline 特征面静压 $(\mathrm{Pa})$ & -36.7 & -49.1 & 12.4 \\
\hline 体积流量 $(\mathrm{m} / \mathrm{s} 3)$ & 16365.0 & 15844.1 & 520.9 \\
\hline
\end{tabular}

无环境风、无消声器阵列时空冷单元流量分布如图 5 所示, 在无风条件下, 空冷平台各单元流量分配较为均匀, 但由于受到平台的 “边界效应” 的影响, 流量分布规律呈 现中间高, 周边低的趋势, 并呈现对称分布。由此, 无风 情况下, 冷却单元流量分配都较为均匀, 增加消声器阵列 后，均有一定的流量损失情况。

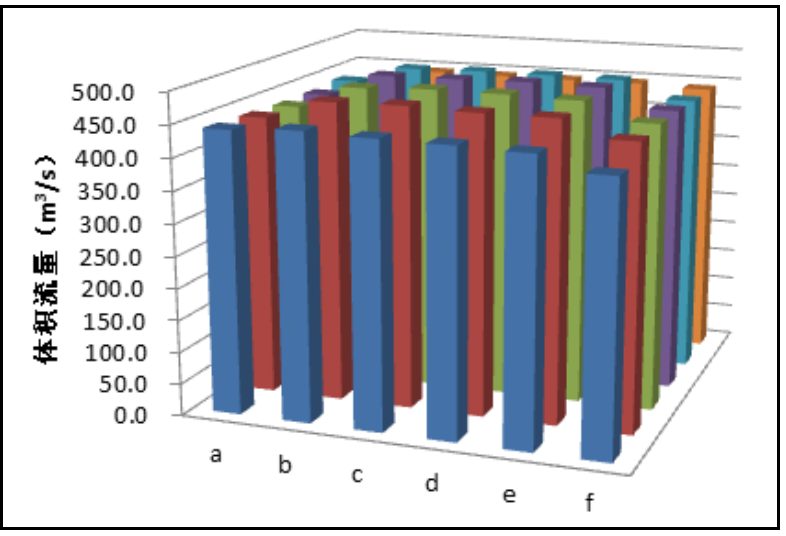

图 5 冷却单元流量分配图 (无消声器\&无环境风)

从图 6 所示的流场示意图中看出, 气流通过消声器阵 列时被整理成从下往上的流动, 流线分布较整齐, 减少了 风机的侧向进气流, 消声器阵列对于空冷平台流场并无较 明显的负面影响，相反起到了一定的导流整流作用。

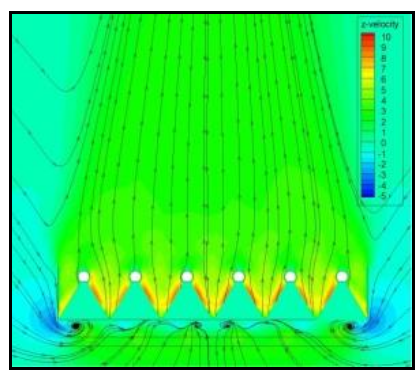

无消声器

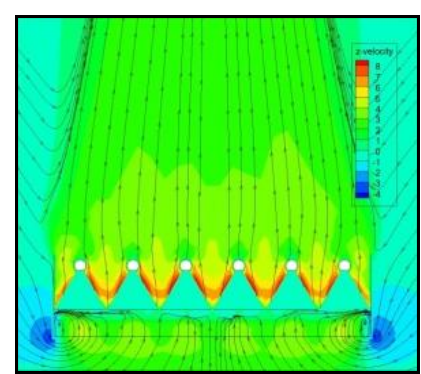

导流片式消声器阵列
图 6 空冷平台 $\mathrm{X}=0$ 截面流场 $(\mathrm{m} / \mathrm{s})$

\section{2 有环境风条件}

在本次计算中暂时忽略风向的影响, 只选择东风方向, 风速为 $2.0 \mathrm{~m} / \mathrm{s} 、 3.5 \mathrm{~m} / \mathrm{s} 、 5.0 \mathrm{~m} / \mathrm{s}$ 。 
由表 2 可以看出, 当环境风速为 $2.0 \mathrm{~m} / \mathrm{s}$ 时, 消声器的 压力损失为 $7.0 \mathrm{~Pa}$, 明显优于无风状态下的压力损失 $12.4 \mathrm{~Pa}$; 当风速为 $3.5 \mathrm{~m} / \mathrm{s} 、 5.0 \mathrm{~m} / \mathrm{s}$ 时, 消声器的压力损失分 别为 $6.5 \mathrm{~Pa} 、 6.8 \mathrm{~Pa}$, 同样小于无风情况下的压力损失。这 表明, 在有环境风的情况下, 消声器阵列对空冷平台的压 力影响有明显减小。

表 2 静压比较

\begin{tabular}{|c|c|c|c|c|}
\hline 特征面静压 & 无风 & 东风 $2.0(\mathrm{~m} / \mathrm{s})$ & 东风 3.5 $(\mathrm{m} / \mathrm{s})$ & 东风 $5.0(\mathrm{~m} / \mathrm{s})$ \\
\hline $\begin{array}{c}\text { 无消声器 } \\
(\mathrm{Pa})\end{array}$ & -36.7 & -49.6 & -55.6 & -59.7 \\
\hline $\begin{array}{c}\text { 有消声器 } \\
(\mathrm{Pa})\end{array}$ & -49.1 & -56.6 & -62.1 & -66.5 \\
\hline $\begin{array}{c}\text { 静压损失 } \\
(\mathrm{Pa})\end{array}$ & 12.4 & 7 & 6.5 & 6.8 \\
\hline
\end{tabular}

由表 3 可以看出, 随着风速的增加, 空冷平台的整体 流量降低, 在风速为 $2.0 \mathrm{~m} / \mathrm{s} 、 3.5 \mathrm{~m} / \mathrm{s}$ 的条件下, 空冷平台 的流量分别为设计流量的 $91.6 \% 、 88.7 \%$, 流量损失分别为 $8.4 \% 、 11.3 \%$; 此时, 消声器对平台造成的损失为 $2.7 \%$ 和 $2.6 \%$, 与无风相差不大。当风速增大到 $5.0 \mathrm{~m} / \mathrm{s}$ 时, 平台流 量有一定程度的降低, 为设计流量的 $84.8 \%$, 流量损失分 别为 $15.2 \%$ 。对比无、有消声器时环境风对空冷平台整体 流量的影响时发现, 当风速达到 $5 \mathrm{~m} / \mathrm{s}$ 时, 消声器阵列对空 冷平台的流量起到了一定的保护作用, 反而减少了环境风 对于空冷平台流量下降的贡献。

表 3 流量比较

\begin{tabular}{|c|c|c|c|c|}
\hline 体积流量 & 无风 & 东风 $2.0(\mathrm{~m} / \mathrm{s})$ & 东风 3.5 $(\mathrm{m} / \mathrm{s})$ & 东风 $5.0(\mathrm{~m} / \mathrm{s})$ \\
\hline $\begin{array}{c}\text { 无消声器 } \\
(\mathrm{m} 3 / \mathrm{s})\end{array}$ & 16365.0 & 16031.2 & 15504.5 & 14754.2 \\
\hline $\begin{array}{c}\text { 有消声器 } \\
(\mathrm{m} 3 / \mathrm{s})\end{array}$ & 15844.1 & 15599.6 & 15108.7 & 14441.7 \\
\hline $\begin{array}{c}\text { 流量损失 } \\
(\mathrm{m} 3 / \mathrm{s})\end{array}$ & 520.9 & 431.6 & 395.8 & 312.5 \\
\hline $\begin{array}{c}\text { 损失比例 } \\
(\%)\end{array}$ & $3.2 \%$ & $2.7 \%$ & $2.6 \%$ & $2.1 \%$ \\
\hline
\end{tabular}

由于环境风的影响, 空冷平台会出现不同程度的热空 气回流现象, 使得进风口温度增加, 从而降低空冷效率。 增加消声器阵列后, 可改善进风口流场的整流效果, 同时 增加了进风口距离, 降低了热空气对风机进口的影响程度, 对热空气回流现象有一定的改善作用。

\section{5. 总结}

本文建立多孔介质等效消声器阵列的局部模型, 通过 对空冷平台流场的仿真计算, 研究了某种消声器阵列结构 对空冷平台性能的影响, 得到如下结论:

(1) 采用多孔介质模型等效消声器阵列进行空冷平台 流场仿真是可行的;

（2）消声器阵列对空冷平台流场特性的影响。

通过对加入消声器阵列空冷平台的流场数值模拟分 析, 为消声器阵列的设计和改进提供数据支持以达到降低 空冷平台噪声的目的。

\section{参考文献(References)}

[1] Bear J. Dynamics of fluids in porous media. Elsevier, NewYork, 1972.

[2] Viswamath $\mathrm{R}$ K at ta, W. M. Roquemore, Study on Trapped-vortex Combustor-effect of Injection on Dynamics of Non-reacting and Reacting Flows in a Cavity. American Institute of Aeronautics and Astronautics 1997.

[3] Yakhot V, Orzag5A. Renormalization group analysis of turbulence: basie theory. J Seient ComPut, 1986(1):3-11.

[4] Patankar S V, Spalding D B•A calculation procedure for the transient and steady state behavior of shell-and-tube heat exchangers $[\mathrm{A}] \cdot$ Afgan N F, Schlunder E V•Heat exchangers: Design and Theary Source Book - New York: McGraw-Hill, 1974•155 176.

[5] Alkam M K. Al-Nimr M A. Transient non-Darcian forced convection flow in a pipe partially filled with a porous material. Int. J. Heat Mass Transfer, 1998, 41(2): 347-356.

[6] P.J. Hotchikss, C.J. Meyer, T.W. von Backstorm. Numerical investigation into the effect of cross flow on the performance of axial flow fans in forced draught air-cooled heat exchangers. Applied Thermal Engineering. 2006, 26. 